\title{
Aptamers in virology: recent advances and challenges
}

\author{
Jennifer M. Binning 1,2 , Daisy W. Leung ${ }^{1}$ and Gaya K. Amarasinghe ${ }^{1 *}$ \\ Department of Pathology and Immunology, Washington University School of Medicine, St. Louis, MO, USA \\ ${ }^{2}$ Biochemistry Graduate Program, Department of Biochemistry, Biophysics and Molecular Biology, lowa State University, Ames, IA, USA
}

\section{Edited by:}

Akio Adachi, University of Tokushima Graduate School, Japan

\section{Reviewed by:}

Mikako Fujita, Kumamoto University, Japan

Yasuyuki Miyazaki, University of

Tokushima Graduate School, Japan

*Correspondence:

Gaya K. Amarasinghe, Department of Pathology and Immunology,

Washington University School of

Medicine, Campus Box 8118, 660

South Euclid Avenue, St. Louis, MO

63110, USA.

e-mail: gamarasinghe@path.wustl.edu
Aptamers generated from randomized libraries of nucleic acids have found utility in a wide variety of fields and in the clinic. Aptamers can be used to target both intracellular and extracellular components, including small molecules, proteins, cells, and viruses. With recent technological developments in stringent selection and rapid isolation strategies, it is likely that aptamers will continue to make an impact as useful tools and reagents. Although many recently developed aptamers are intended for use as therapeutic and diagnostic agents, use of aptamers for basic research, including target validation, remains an active area with high potential to impact our understanding of molecular mechanisms and for drug discovery. In this brief review, we will discuss recent aptamer discoveries, their potential role in structural virology, as well as challenges and future prospects.

Keywords: aptamer, therapeutic and diagnostic agents, structural virology, research tools

\section{INTRODUCTION}

Aptamers are in vitro selected nucleic acid molecules that bind to a broad range of targets with high affinity and specificity. The basic isolation process, termed "systematic evolution of ligands by exponential enrichment" or SELEX (Ellington and Szostak, 1990; Robertson and Joyce, 1990; Tuerk and Gold, 1990), utilizes a large initial pool of randomized nucleic acids from which high affinity binders are isolated and enriched through subsequent rounds of selection (Figure 1). Different in vitro isolation strategies can be used to generate aptamers against many diverse targets under different selection and counter selection conditions, leading to facile identification of aptamers that bind targets ranging from a few atoms (small molecules) to many atoms (macromolecules) to intact cells and viruses. Most aptamers characterized to date are single stranded DNA or RNA molecules ranging from 20 to 90 bases, although longer aptamers have been generated. These single stranded polynucleotide sequences can fold into a variety of secondary structural elements, including double stranded RNA (dsRNA) or dsDNA, stem loops, pseudoknots, kinks, and bulges, providing multiple recognition surfaces for target binding.

Aptamers are often compared to antibodies, due to the high specificity for their targets generated through stringent in vitro selection. However, aptamers have notable advantages over antibodies, including low immunogenicity and the potential for chemical derivatization to enhance in vivo stability and bioavailability (Jellinek et al., 1995; Chelliserrykattil and Ellington, 2004; Kato et al., 2005). The aptamer selection process can be carried out in as little as $2-4$ weeks, which is significantly shorter compared to antibody development (months). Since the initial description of the SELEX process over two decades ago, general principles guiding the selection process have remained largely unaltered. However, modifications to the original process now allow for highly efficient and extremely stringent selection strategies to be employed against a diverse array of targets. Identified aptamers can be further optimized using biased libraries that result in aptamer subpopulations with higher specificity and/or affinity and other desirable characteristics, such as membrane permeability. Coupling SELEX methods with other techniques, such as capillary electrophoresis and surface plasmon resonance (SPR), can reduce the number of selection rounds and shorten the period for aptamer discovery against a given target. In addition, facile generation of modified aptamers allows for coupling of diagnostic tags, which can extend the use of aptamers through prolonged lifetimes in serum. Given the traditional comparisons with antibodies, the utility of aptamers in the clinic is taking longer than anticipated from the outset of discovery. Nonetheless, with the 2004 FDA approval of pegaptanib, an aptamer-based drug used to treat wet age-related muscular degeneration, and others in the pipeline, it is clear that aptamers will continue to contribute to our understanding of normal and aberrant cellular processes as well as disease treatment. Recent comprehensive reviews highlight advances in basic research (James, 2001, 2007; Syed and Pervaiz, 2010; Sun et al., 2011) and clinical settings (Bunka and Stockley, 2006; Gopinath, 2007, 2008; Meyer et al., 2011). These developments have positioned aptamers to make a significant impact in many areas.

\section{APTAMERS AND VIRUSES}

The host-viral interface has become an increasingly popular target for aptamers because interactions between nucleic acids and proteins are critical to viral replication (Figure 2). Although not a prerequisite, targeting nucleic acid binding proteins by aptamers has a high probability of success due to the presence of basic surface charges on most nucleic acid binding proteins. Below we summarize select studies in order to highlight the use of aptamers against viral components and discuss challenges and future prospects of the field. 


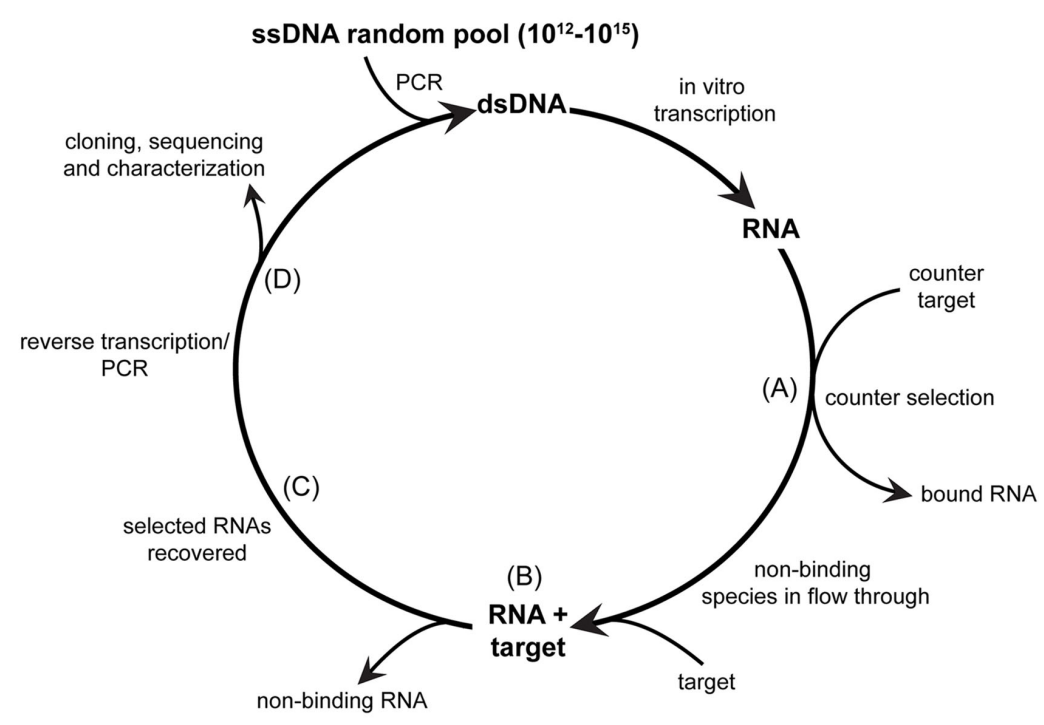

FIGURE 1 |The SELEX process. An initial pool of dsDNA is in vitro transcribed. The resulting pool of RNA is subjected to (A) counter selection and/or (B) selection by filter binding. (C) Selected RNA is recovered,

reverse transcribed, and amplified by PCR. (D) The resulting RNA pool is subjected to another round of SELEX for enrichment or cloned and sequenced.

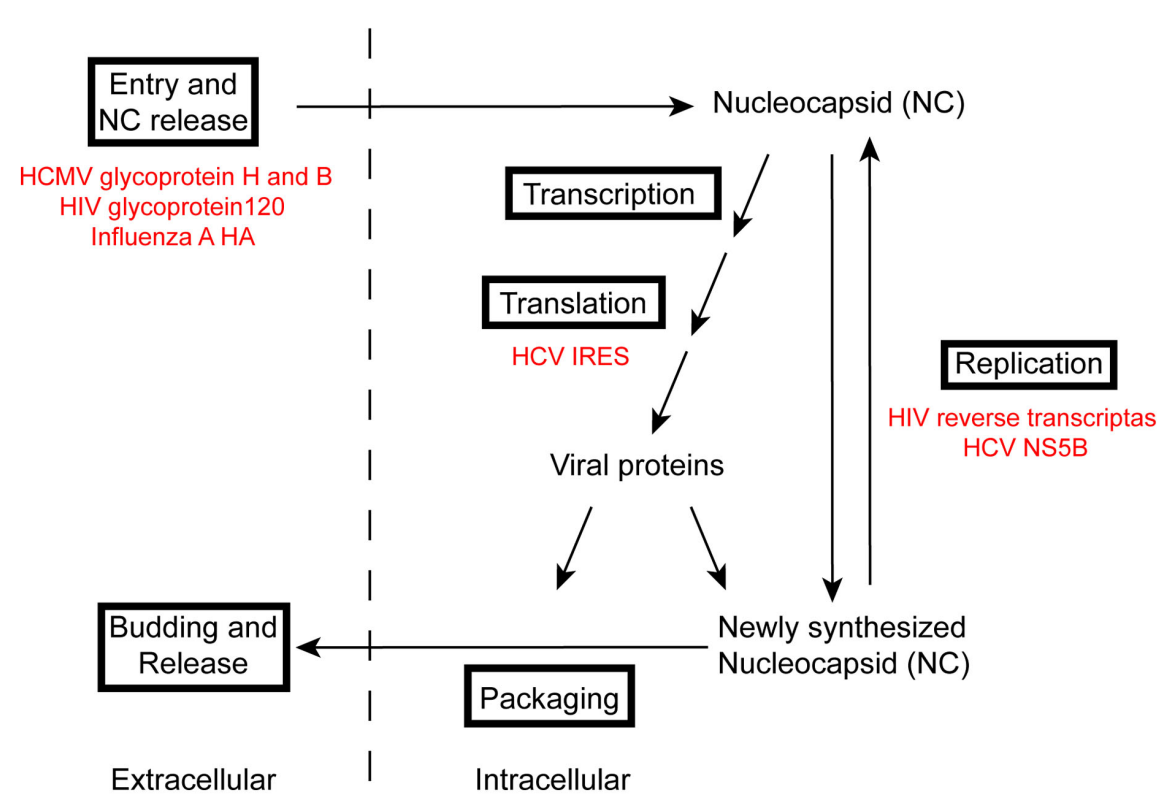

FIGURE 2 | Key steps in the viral replication cycle are targets for aptamer development. Examples of viral proteins targeted by aptamers are identified in red.

\section{APTAMERS IN THERAPEUTICS}

Aptamers provide an alternative method for the development of therapeutic agents to counter viral infections. Aptamers can be used to inhibit viral infectivity at any stage in the viral replication cycle, including viral entry, which has the potential to prevent initial infection. The development of aptamers against the human immunodeficiency virus HIV-1 has been of interest for drug discovery. In order to target HIV entry into helper T-cells, aptamers generated against HIV glycoprotein 120 (gp120) competes with the HIV co-receptor, CCR5. The aptamer binding site is located in a highly conserved region of gp120, allowing neutralization of a broad range of HIV isolates by disrupting the gp120-CCR5 interaction (Dey et al., 2005a,b). Binding to a conserved region of gp120 is critical due to the propensity of HIV to undergo mutagenesis that results in drug-resistance. The antigp120 aptamer has not been approved for clinical trials, but has 
been used as a basis for developing chimeric aptamers (Neff et al., 2011; Wheeler et al., 2011; Zhou et al., 2011). Coupling of siRNA and the anti-gp120 aptamer through phi29 packaging RNA combines two functions (Zhou et al., 2011). The anti-gp120 aptamer has an inhibitory effect, while siRNA downregulates the expression of its target.

Aptamer generation against human cytomegalovirus (HCMV) particles was carried out without prior information on specific targets (Wang et al., 2000). Two aptamers, L13 and L19, were isolated that inhibit HCMV plaque formation and growth. The antiviral activity of L13 and L19 aptamers identified HCMV glycoproteins $\mathrm{B}$ and $\mathrm{H}$ as targets, respectively, by binding to the glycoproteins and blocking viral entry.

Viral RNA-dependent RNA polymerases have been a common target because viral polymerases use RNA as the template during genome replication. For example, the hepatitis $\mathrm{C}$ virus (HCV) polymerase non-structural protein $5 \mathrm{~B}$ (NS5B), is required for transcribing the HCV genome and has been used to generate both anti-NS5B RNA and DNA aptamers (Biroccio et al., 2002; Bellecave et al., 2003, 2008). Both sets of aptamers have been shown to bind to NS5B with high affinity and inhibit polymerase activity. The mechanism of inhibition of the anti-NS5B RNA aptamer is non-competitive with regards to the template RNA, and mutagenesis studies revealed that a basic patch within the thumb domain of NS5B is the aptamer binding site (Biroccio et al., 2002). Only one anti-NS5B DNA aptamer inhibits replication by competing with the RNA template; the other inhibits through a non-competitive mechanism in which initiation and post initiation events are disrupted. RNA aptamers capable of inhibiting NS5B polymerase activity were further optimized through the use of deletion clones and point mutagenesis, which identified a GC-rich motif and a stem-loop with a bulge as important features for aptamer binding to NS5B (Kanamori et al., 2009).

In the context of HIV pathogenesis, viral reverse transcriptase (RT) converts the single stranded RNA (ssRNA) viral genome into dsDNA. RNA aptamers that target HIV-RT effectively inhibit the synthesis of cDNA (Tuerk et al., 1992). The ligand 1.1 aptamer targeting HIV-RT is postulated to reduce adverse side effects, a main concern with current HIV-RT drugs, such as 3'-azido-3'deoxythymidine (AZT) and dideoxyinosine. Moreover, by targeting an enzyme early in the HIV-1 replication cycle, the likelihood that mutations will arise resulting in drug-resistance should diminish. These factors make aptamers generated against HIV-RT promising candidates for drug development.

Inhibition of protein translation is a key host defense mechanism against viral infections since viruses are completely dependent on the host machinery for protein synthesis. However, viruses have developed various means to evade host defenses and hijack the host translational machinery. For example, the HCV mRNA has an internal ribosome entry site (IRES), a structured region within the mRNA that binds the ribosome and initiates cap-independent translation, allowing HCV to circumvent the requirement for host initiation and elongation factors. Aptamers targeting the HCV IRES inhibit IRES-dependent translation of HCV proteins (Aldaz-Carroll et al., 2002; Kikuchi et al., 2003, 2005; Da Rocha Gomes et al., 2004). Furthermore, many of these HCV IRES aptamers inhibit translation both in vitro and in vivo, highlighting the potential therapeutic value of these aptamers since they target RNA secondary structure that is characteristic of select viral mRNAs but not host mRNA. Therefore, such selection may result in a reduction of negative side effects. The viral inhibitory properties of HCV anti-IRES aptamers can be enhanced through conjugation to other aptamers, which target different binding sites within the HCV IRES. This results in a conjugated anti-IRES aptamer that binds the HCV IRES with a higher affinity and is functionally more effective than the unconjugated aptamers (Kikuchi et al., 2009). Alternatively, HCV anti-IRES aptamers can be coupled to the hammerhead ribozyme to prevent translation. The chimeric RNA molecule, HH363-50, inhibits IRES-dependent translation in vitro likely through disruption of $80 \mathrm{~S}$ complex formation with no effect on IRES-independent translation. HH363-50 also reduced the level of HCV RNA due to the ribozyme activity of the molecule (Romero-Lopez et al., 2009).

\section{APTAMERS IN VIRAL DIAGNOSTICS}

Early and reliable detection of pathogens is a critical step in the successful treatment of infection. Due to many potential advantages aptamers provide, aptamers are also ideal tools for diagnostics. Many examples of aptamers as a detection tool have been described. These studies include simple modifications to the enzyme linked immunosorbent assay (ELISA) as well as more complex diagnostic systems, such as those that use inhibitory aptamers to suppress multi turnover enzymes (Zhou et al., 2010).

The Influenza A virus hemagglutinin (HA) and neuraminidase (NA) antigens are glycoproteins found on the surface of the viral particle, and serve important roles in host membrane fusion. A number of aptamers have been isolated against Influenza A HA that inhibit viral infectivity (Jeon et al., 2004; Misono and Kumar, 2005; Gopinath et al., 2006; Dhar and Datta, 2009; Park et al., 2011). In addition to disrupting viral HA-mediated membrane fusion, the P30-10-16 aptamer can distinguish between closely related Influenza A strains. This aptamer property is novel, considering that most monoclonal antibodies against HA have been unsuccessful at differentiating among influenza subtypes. Although initial studies were carried out by SPR-based detection, coupling of these aptamers to fluorophores or other signaling molecules could provide facile detection of virus subtypes. Development of these aptamers for viral subtype diagnostics will provide a significant advancement in our ability to differentiate highly pathogenic influenza strains from those that are less virulent and will likely yield important insights into its mechanism of action.

The Hepatitis B virus (HBV) surface antigen (HBsAg) is found on the membrane of HBV-infected hepatocytes. A fluorescently labeled aptamer targeting HBsAg, HBs-A22, was used to isolate cells expressing HBsAg. Use of fluorescence microscopy showed that that anti-HBsAg aptamer bound to the HBsAg-positive cell line (HepG2.2.15) but not to the HBsAg-negative cell line (HepG2). These results establish the use of aptamers for imaging, but also provide the first HBV specific antigen aptamer that could be used for early detection and treatment of HBV-infected cells (Liu et al., 2010).

Aptamers can also function as diagnostic tools when coupled to existing biosensors (Davis et al., 1998; Jhaveri et al., 2000; 
Fang et al., 2001; O’Sullivan, 2002). Biosensors utilize a biological recognition element, such as aptamers, and a transducer for easier detection and quantification. An aptamer-based biosensor, or aptasensor, was isolated against the trans-activator of transcription (Tat) of HIV-1, which is important for regulating the early phases of HIV-1 infection (Chang et al., 1997; Mucha et al., 2002). The Tat aptasensor was generated by immobilizing the aptamer on a piezoelectric quartz-crystal, the transduction component of the aptasensor. Comparison of the Tat aptasensor to a corresponding immunosensor that uses an anti-Tat monoclonal antibody showed that the two biosensors are similar in terms of sensitivity and reproducibility (Minunni et al., 2004).

A multicomponent reporter system consisting of an inhibitory aptamer bound to a restriction endonuclease and a target complement/trigger system was successful in differentiating Dengue virus serotypes (Fletcher et al., 2010). One of the key advantages of this method is that the same aptamer/enzyme complex can be used since only the nucleic acid complement/trigger needs modification to detect new targets. Such a system can be more sensitive due to the multi-turnover nature of the endonuclease. Further development of these aptasensors and incorporating more stable chemical derivatives will likely overcome some of the limitations of current aptasensors.

\section{APTAMERS IN BASIC RESEARCH}

In addition to diagnostic and therapeutic applications, aptamers can be used as laboratory reagents in a number of biochemical and cell-based assays, similar to antibodies. Aptamers have key advantages over antibodies in that they often have higher affinity and specificity for their targets with some binding constants $\left(K_{\mathrm{D}}\right)<1 \mathrm{nM}$, which results in an enhanced signal-to-noise ratio. Additionally, aptamers can be generated against almost any target ranging from small organic molecules to proteins to whole cells (Jayasena, 1999), and they are often times smaller than antibodies (7-30 kDa for aptamers vs. $\sim 150 \mathrm{kDa}$ for antibodies) allowing them to bind regions inaccessible to antibodies.

By utilizing aptamers that disrupt protein-protein interactions, one can begin to dissect and characterize cellular pathways. The human T-cell leukemia virus type 1 Tax protein is a trans-activator that regulates the expression of various viral and cellular genes. Tax cannot directly bind DNA in the absence of certain host transcription factors, suggesting that protein-protein interactions are essential for Tax function. The YT1 aptamer disrupts Tax interactions with the cyclic AMP-response element binding protein (CREB) and NF- $\kappa \mathrm{B}$, but not serum response factor (SRF), all of which are known to interact with Tax proteins (Tian et al., 1995). These results suggest that the CREB and NF- $\kappa \mathrm{B}$ binding interfaces on Tax may potentially overlap or are in close proximity, whereas the SRF binding site occupies a different surface on Tax. Therefore, aptamers provide a potential tool to map interaction surfaces between binding partners.

Assembly of the viral nucleocapsid (NC) requires multiple protein-protein and protein-nucleic acid interactions. The HIV $\mathrm{NC}$ is a highly conserved protein that plays a key role in RNA encapsidation and viral replication, and specifically binds viral genomic RNA through the psi sequence. An aptamer generated against $\mathrm{NC}$ competes with the psi sequence and prevents proper encapsidation of the viral genome, which likely inhibits proper packaging of the genomic RNA (Kim et al., 2002). Other HIV$1 \mathrm{NC}$ aptamers against the HIV-1 Gag protein bind the matrix and the NC domains of Gag and reduced HIV replication in cultured cells. The inhibitory effect of these aptamers was not due to a defect in virion release but the downregulation of intracellular Gag protein and mRNA. A subset of aptamers which specifically bound NC competes with the psi packing signal of HIV-1, and disrupts interactions between Gag and viral RNA (Ramalingam et al., 2011). Unlike other aptamers that inhibit early stages in viral infection, the $\mathrm{NC}$ aptamer targets events that occur after viral entry, and therefore, allows one to study the intracellular effects of viral infection.

Using aptamers to specifically target and modulate functions of proteins within cellular pathways or the viral replication cycle can provide invaluable information about these respective systems. Nonetheless, an additional advantage of using aptamers to investigate cellular signaling is the ability to selectively turn the aptamers "on" and "off" with effectors. Further advances in the spatial and temporal regulation of aptamers will be important for enhancing our understanding of viral/host interactions. The potential regulation of aptamers with effector molecules is highlighted by Vuyisich and Beal (2002) in the regulation of formamidopyrimidine glycosylase (Fpg), a bacterial enzyme involved in DNA repair. A modified SELEX protocol was used where RNA-bound Fpg was treated with neomycin in order to elute the bound RNA. The Fpg aptamer inhibited Fpg activity, but the functionality was restored in the presence of neomycin. Rusconi et al. (2002) utilized antisense RNA in order to regulate the function of an aptamer targeted against the blood coagulation factor IXa. The anti-sense RNA base pairs to complementary regions within the aptamer and disrupts aptamer function. The factor IXa aptamer and anti-sense RNA antidote pair was an effective, reversible anticoagulant and shows potential for clinical application.

\section{FUTURE PROSPECTS}

Many viral proteins bind nucleic acids, although not a required characteristic, makes viruses good targets for aptamer development. As outlined above, aptamers have been used in a variety of studies to characterize and counter host-viral interactions, including targeting individual viral components or whole cells. A significant advantage of the aptamer technology lies in its ability to identify aptamers against a given target without a priori knowledge of the exact site or target. With limited information on the binding site, aptamers can be generated toward a given target and subsequently tested for regulatory activity either in vitro or in vivo. Similarly, aptamers can be used in target validation, where judicious use of aptamers can provide distinct advantages. Aptamers can also be used to dissect protein-protein interfaces and to validate traditional targets for small molecules, such as enzyme active sites. Current selection of small molecules toward known targets often depend on a variety of factors, including the availability of validating functional assays, structural and biochemical data, prior to initiating drug discovery efforts, which consume large amounts of time and resources. In contrast, one can develop aptamers with a fraction of the cost and time in a laboratory setting. 
It is interesting to note that most aptamers form unusual and dynamic conformations that can potentially allow aptamers to target protein-protein, protein-nucleic acid, and small molecule interactions. However, in many of the examples reviewed, there is limited knowledge on the structural and biochemical aspects of these aptamer/target interactions, and available data are primarily restricted to secondary structure predictions or mapping. The addition of structural and biochemical studies of aptamer/target pairs can enhance the utility of these aptamers by providing new information on the aptamer/target interfaces and by providing

\section{REFERENCES}

Aldaz-Carroll, L., Tallet, B., Dausse, E., Yurchenko, L., and Toulme, J. J. (2002). Apical loop-internal loop interactions: a new RNARNA recognition motif identified through in vitro selection against RNA hairpins of the hepatitis C virus mRNA. Biochemistry 41, 5883-5893.

Bellecave, P., Andreola, M. L., Ventura, M., Tarrago-Litvak, L., Litvak, S., and Astier-Gin, T. (2003). Selection of DNA aptamers that bind the RNA-dependent RNA polymerase of hepatitis $\mathrm{C}$ virus and inhibit viral RNA synthesis in vitro. Oligonucleotides 13, 455-463.

Bellecave, P., Cazenave, C., Rumi, J., Staedel, C., Cosnefroy, O., Andreola, M. L., Ventura, M., Tarrago-Litvak, L., and Astier-Gin, T. (2008). Inhibition of hepatitis $\mathrm{C}$ virus (HCV) RNA polymerase by DNA aptamers: mechanism of inhibition of in vitro RNA synthesis and effect on HCVinfected cells. Antimicrob. Agents Chemother. 52, 2097-2110.

Biroccio, A., Hamm, J., Incitti, I., De Francesco, R., and Tomei, L. (2002). Selection of RNA aptamers that are specific and high-affinity ligands of the hepatitis $\mathrm{C}$ virus RNAdependent RNA polymerase. J. Virol. 76, 3688-3696.

Bunka, D. H., and Stockley, P. G. (2006). Aptamers come of age - at last. Nat. Rev. Microbiol. 4, 588-596.

Chang, H. C., Samaniego, F., Nair, B. C., Buonaguro, L., and Ensoli, B. (1997). HIV-1 Tat protein exits from cells via a leaderless secretory pathway and binds to extracellular matrixassociated heparan sulfate proteoglycans through its basic region. AIDS 11, 1421-1431.

Chelliserrykattil, J., and Ellington, A. D. (2004). Evolution of a T7 RNA polymerase variant that transcribes $2^{\prime}$ O-methyl RNA. Nat. Biotechnol. 22, 1155-1160.

Da Rocha Gomes, S., Dausse, E., and Toulme, J. J. (2004). Determinants of apical loop-internal loop RNARNA interactions involving the $\mathrm{HCV}$
IRES. Biochem. Biophys. Res. Commun. 322, 820-826.

Davis, K. A., Lin, Y., Abrams, B., and Jayasena, S. D. (1998). Staining of cell surface human CD4 with $2^{\prime}$-F-pyrimidine-containing RNA aptamers for flow cytometry. Nucleic Acids Res. 26, 3915-3924.

Dey, A. K., Griffiths, C., Lea, S. M., and James, W. (2005a). Structural characterization of an anti-gp120 RNA aptamer that neutralizes R5 strains of HIV-1. RNA 11, 873-884.

Dey, A. K., Khati, M., Tang, M., Wyatt, R., Lea, S. M., and James, W. (2005b). An aptamer that neutralizes R5 strains of human immunodeficiency virus type 1 blocks gp120-CCR5 interaction. J. Virol. 79, 13806-13810.

Dhar, G. S., and Datta, A. (2009). Targeting pseudoknots in $\mathrm{H} 5 \mathrm{~N} 1$ hemagglutinin using designed aptamers. Bioinformation 4, 193-196.

Ellington, A. D., and Szostak, J. W. (1990). In vitro selection of RNA molecules that bind specific ligands. Nature 346, 818-822.

Fang, X., Cao, Z., Beck, T., and Tan, W. (2001). Molecular aptamer for realtime oncoprotein platelet-derived growth factor monitoring by fluorescence anisotropy. Anal. Chem. 73, 5752-5757.

Fletcher, S. J., Phillips, L. W., Milligan, A. S., and Rodda, S. J. (2010). Toward specific detection of Dengue virus serotypes using a novel modular biosensor. Biosens. Bioelectron. 26, 1696-1700.

Gopinath, S. C. (2007). Antiviral aptamers. Arch. Virol. 152, 2137-2157.

Gopinath, S. C. (2008). Anti-coagulant aptamers. Thromb. Res. 122, 838-847.

Gopinath, S. C., Misono, T. S., Kawasaki, K., Mizuno, T., Imai, M., Odagiri, T., and Kumar, P. K. (2006). An RNA aptamer that distinguishes between closely related human influenza viruses and inhibits haemagglutinin-mediated membrane fusion. J. Gen. Virol. 87, 479-487.

information for structure-based optimization of aptamers. Continual development and optimization of multifunctional, chimeric aptamers, and aptamer libraries for strain specific viral identification will lead to more broadly applicable therapeutic and diagnostic applications in the future.

\section{ACKNOWLEDGMENTS}

Work in our laboratory is supported by NIH grants [AI089547 to Gaya K. Amarasinghe (CF Basler-PI) and AI059536]. We thank P. Ramanan for critical reading of the manuscript.

James, W. (2001). Nucleic acid and polypeptide aptamers: a powerful approach to ligand discovery. Curr. Opin. Pharmacol. 1, 540-546.

James, W. (2007). Aptamers in the virologists' toolkit. J. Gen. Virol. 88, 351-364.

Jayasena, S. D. (1999). Aptamers: an emerging class of molecules that rival antibodies in diagnostics. Clin. Chem. 45, 1628-1650.

Jellinek, D., Green, L. S., Bell, C., Lynott, C. K., Gill, N., Vargeese, C., Kirschenheuter, G., Mcgee, D. P., Abesinghe, P., Pieken, W. A., Shapiro, R., Rifkin, D. B., Moscatelli, D., and Janjic, N. (1995). Potent 2'-amino$2^{\prime}$-deoxypyrimidine RNA inhibitors of basic fibroblast growth factor. Biochemistry 34, 11363-11372.

Jeon, S. H., Kayhan, B., Ben-Yedidia, T., and Arnon, R. (2004). A DNA aptamer prevents influenza infection by blocking the receptor binding region of the viral hemagglutinin. J. Biol. Chem. 279, 48410-48419.

Jhaveri, S., Rajendran, M., and Ellington, A. D. (2000). In vitro selection of signaling aptamers. Nat. Biotechnol. 18, 1293-1297.

Kanamori, H., Yuhashi, K., Uchiyama, Y., Kodama, T., and Ohnishi, S. (2009). In vitro selection of RNA aptamers that bind the RNAdependent RNA polymerase of hepatitis $\mathrm{C}$ virus: a possible role of GC-rich RNA motifs in NS5B binding. Virology 388, 91-102.

Kato, Y., Minakawa, N., Komatsu, Y., Kamiya, H., Ogawa, N., Harashima, H., and Matsuda, A. (2005). New NTP analogs: the synthesis of $4^{\prime}$ thioUTP and $4^{\prime}$-thioCTP and their utility for SELEX. Nucleic Acids Res. 33, 2942-2951.

Kikuchi, K., Umehara, T., Fukuda, K., Hwang, J., Kuno, A., Hasegawa, T., and Nishikawa, S. (2003). RNA aptamers targeted to domain II of hepatitis $C$ virus IRES that bind to its apical loop region. J. Biochem. 133, 263-270.

Kikuchi, K., Umehara, T., Fukuda, K., Kuno, A., Hasegawa, T., and
Nishikawa, S. (2005). A hepatitis $\mathrm{C}$ virus (HCV) internal ribosome entry site (IRES) domain III-IVtargeted aptamer inhibits translation by binding to an apical loop of domain IIId. Nucleic Acids Res. 33, 683-692.

Kikuchi, K., Umehara, T., Nishikawa, F., Fukuda, K., Hasegawa, T., and Nishikawa, S. (2009). Increased inhibitory ability of conjugated RNA aptamers against the HCV IRES. Biochem. Biophys. Res. Commun. 386, 118-123.

Kim, S. J., Kim, M. Y., Lee, J. H., You, J. C. and Jeong, S. (2002). Selection and stabilization of the RNA aptamers against the human immunodeficiency virus type-1 nucleocapsid protein. Biochem. Biophys. Res. Commun. 291, 925-931.

Liu, J., Yang, Y., Hu, B., Ma, Z. Y., Huang, H. P., Yu, Y., Liu, S. P., Lu, M. J., and Yang, D. L. (2010). Development of HBsAg-binding aptamers that bind HepG2.2.15 cells via HBV surface antigen. Virol. Sin. 25, 27-35.

Meyer, C., Hahn, U., and Rentmeister, A. (2011). Cell-specific aptamers as emerging therapeutics. J. Nucleic Acids 2011, 904750.

Minunni, M., Tombelli, S., Gullotto, A., Luzi, E., and Mascini, M. (2004). Development of biosensors with aptamers as bio-recognition element: the case of HIV-1 Tat protein. Biosens. Bioelectron. 15, 1149-1156.

Misono, T. S., and Kumar, P. K. (2005). Selection of RNA aptamers against human influenza virus hemagglutinin using surface plasmon resonance. Anal. Biochem. 342, 312-317.

Mucha, P., Szyk, A., Rekowski, P., and Barciszewski, J. (2002). Structural requirements for conserved Arg52 residue for interaction of the human immunodeficiency virus type 1 trans-activation responsive element with trans-activator of transcription protein (49-57). Capillary electrophoresis mobility shift assay. J. Chromatogr. A 968, 211-220. 
Neff, C. P., Zhou, J., Remling, L., Kuruvilla, J., Zhang, J., Li, H., Smith, D. D., Swiderski, P., Rossi, J. J., and Akkina, R. (2011). An aptamersiRNA chimera suppresses HIV-1 viral loads and protects from helper $\mathrm{CD} 4(+) \mathrm{T}$ cell decline in humanized mice. Sci. Transl. Med. 3, 66ra66.

O’Sullivan, C. K. (2002). Aptasensors - the future of biosensing? Anal. Bioanal. Chem. 372, 44-48.

Park, S. Y., Kim, S., Yoon, H., Kim, K. B., Kalme, S. S., Oh, S., Song, C. S., and Kim, D. E. (2011). Selection of an antiviral RNA aptamer against hemagglutinin of the subtype H5 avian influenza virus. Nucleic Acid Ther. 21, 395-402.

Ramalingam, D., Duclair, S., Datta, S. A., Ellington, A., Rein, A., and Prasad, V. R. (2011). RNA aptamers directed to human immunodeficiency virus type $1 \mathrm{Gag}$ polyprotein bind to the matrix and nucleocapsid domains and inhibit virus production. J. Virol. 85, 305-314.

Robertson, D. L., and Joyce, G. F. (1990). Selection in vitro of an RNA enzyme that specifically cleaves single-stranded DNA. Nature 344, 467-468.

Romero-Lopez, C., Diaz-Gonzalez, R., Barroso-Deljesus, A., and
Berzal-Herranz, A. (2009). Inhibition of hepatitis $\mathrm{C}$ virus replication and internal ribosome entry site-dependent translation by an RNA molecule. J. Gen. Virol. 90, 1659-1669.

Rusconi, C. P., Scardino, E., Layzer, J., Pitoc, G. A., Ortel, T. L., Monroe, D., and Sullenger, B. A. (2002). RNA aptamers as reversible antagonists of coagulation factor IXa. Nature 419, 90-94.

Sun, W., Du, L., and Li, M. (2011). Advances and perspectives in cellspecific aptamers. Curr. Pharm. Des. 17, 80-91.

Syed, M. A., and Pervaiz, S. (2010). Advances in aptamers. Oligonucleotides 20, 215-224.

Tian, Y., Adya, N., Wagner, S., Giam, C. Z., Green, M. R., and Ellington, A. D. (1995). Dissecting protein:protein interactions between transcription factors with an RNA aptamer. $R N A$ 1, 317-326.

Tuerk, C., and Gold, L. (1990). Systematic evolution of ligands by exponential enrichment: RNA ligands to bacteriophage T4 DNA polymerase. Science 249, 505-510.

Tuerk, C., Macdougal, S., and Gold, L. (1992). RNA pseudoknots that inhibit human immunodeficiency virus type 1 reverse transcriptase. Proc. Natl. Acad. Sci. U.S.A. 89, 6988-6992.

Vuyisich, M., and Beal, P. A. (2002). Controlling protein activity with ligand-regulated RNA aptamers. Chem. Biol. 9, 907-913.

Wang, J., Jiang, H., and Liu, F. (2000). In vitro selection of novel RNA ligands that bind human cytomegalovirus and block viral infection. RNA 6 , 571-583.

Wheeler, L. A., Trifonova, R., Vrbanac, V., Basar, E., Mckernan, S., Xu, Z., Seung, E., Deruaz, M., Dudek, T., Einarsson, J. I., Yang, L., Allen, T. M., Luster, A. D., Tager, A. M., Dykxhoorn, D. M., and Lieberman, J. (2011). Inhibition of HIV transmission in human cervicovaginal explants and humanized mice using CD4 aptamersiRNA chimeras. J. Clin. Invest. 121, 2401-2412.

Zhou, J., Battig, M. R., and Wang, Y. (2010). Aptamer-based molecular recognition for biosensor development. Anal. Bioanal. Chem. 398, 2471-2480.

Zhou, J., Shu, Y., Guo, P., Smith, D. D., and Rossi, J. J. (2011).
Dual functional RNA nanoparticles containing phi29 motor pRNA and anti-gp120 aptamer for cell-type specific delivery and HIV-1 inhibition. Methods 54, 284-294.

Conflict of Interest Statement: The authors declare that the research was conducted in the absence of any commercial or financial relationships that could be construed as a potential conflict of interest.

Received: 05 January 2012; accepted: 17 January 2012; published online: 08 February 2012.

Citation: Binning JM, Leung DW and Amarasinghe GK (2012) Aptamers in virology: recent advances and challenges. Front. Microbio. 3:29. doi: 10.3389/fmicb.2012.00029

This article was submitted to Frontiers in Virology, a specialty of Frontiers in Microbiology.

Copyright (c) 2012 Binning, Leung and Amarasinghe. This is an open-access article distributed under the terms of the Creative Commons Attribution Non Commercial License, which permits noncommercial use, distribution, and reproduction in other forums, provided the original authors and source are credited. 